\title{
Determinação do prazo de validade do medicamento carbocisteína xarope através do método de Arrhenius
}

\author{
Josélia Larger Manfio ${ }^{1 *}$, Alexandre Dal'Maso' ${ }^{1}$ Ana Maria Pugens' ${ }^{1}$, \\ Liberato Brum Junior ${ }^{1}$, Martin Steppe ${ }^{2}$ \\ ${ }^{1}$ Biocinese - Centro de Estudos Biofarmacêuticos, Toledo, Paraná, ${ }^{2}$ Faculdade de Farmácia, \\ Universidade Federal do Rio Grande do Sul
}

*Correspondência:

J. L. Manfio

Biocinese - Centro de Estudos

Biofarmacêuticos

Rua Ministro Cirne de Lima, 1541

85903-590 - Toledo - PR, Brasil

E-mail: manfiojoselia@bol.com.br
A carbocisteina é um agente mucolitico utilizado como adjuvante no tratamento de infecções do trato respiratório. A qualidade, segurança e eficácia do medicamento durante o seu prazo de validade são responsabilidades da indústria farmacêutica. A validade pode ser determinada através de estudos de estabilidade acelerados, nos quais fatores extrínsecos provocam a degradação do produto. De acordo com Arrhenius, existe uma relação entre temperatura e cinética química. Desta forma, amostras do produto foram expostas a condições drásticas: $40,50,60$ e $70{ }^{\circ} \mathrm{C}$. O método de doseamento do xarope de carbocisteína foi validado podendo ser aplicado nas avaliações de rotina do controle de qualidade e no estudo de estabilidade deste produto. O prazo de validade proposto para o xarope de carbocisteina, calculado através da equação de Arrhenius, para a temperatura de $25{ }^{\circ} \mathrm{C}$ foi de 240,9 dias. No entanto, foi observada diferença entre o prazo proposto e a validade usualmente praticada para este produto. Este estudo, ainda, demonstrou a presença de picos endógenos, que precisam ser melhor estudados a fim de se confirmar a presença de produtos de degradação.

\section{INTRODUÇÃO}

Nas últimas décadas, a avaliação da estabilidade de produtos farmacêuticos tem recebido especial atenção tanto pelos órgãos fiscalizadores quanto pelas empresas produtoras. É dever do fabricante assegurar a qualidade do produto, o que implica conhecimento do mesmo garantido através de criteriosa pesquisa.

A literatura descreve várias maneiras de avaliar a estabilidade de um produto farmacêutico. Considerando os principais fatores responsáveis pela degradação de um produto farmacêutico é possível acelerar esta degradação visando estipular o prazo de validade do produto, ou seja, período de vida útil (Bakshi, Singh, 2002; Lachman, 2001). Apesar dos avanços tecnológicos no estudo de estabilidade dos produtos farmacêuticos, a legislação sanitária brasileira vigente reconhece a avaliação da estabilidade de um produto líquido, envasado em embalagem semi-perme- 
ável, realizado a $40{ }^{\circ} \mathrm{C} \pm 2{ }^{\circ} \mathrm{C}$ e $75 \% \pm 5 \%$ de umidade durante 180 dias como indicativos do prazo de validade provisório. A validade definitiva é confirmada somente através do estudo de estabilidade de longa duração, em que as amostras são mantidas a $30{ }^{\circ} \mathrm{C} \pm 2{ }^{\circ} \mathrm{C}$ e $75 \% \pm 5 \%$ de umidade e analisadas durante o período de validade proposto (Brasil, 2005).

A carbocisteína é comercializada nas formas de xarope e solução oral, não necessitando de prescrição médica para sua dispensação. Oficialmente, existem no Brasil, 38 registros deste medicamento (ANVISA, 2007). Seu efeito mucolítico deve-se ao grupo tiólico livre, que atua diretamente sobre as mucoproteínas, abrindo as ligações dissulfeto e diminuindo a viscosidade do muco. Também tem a capacidade de aumentar a síntese da sialomucina, constituinte fundamental do muco brônquico, de que depende a propriedade reológica do mesmo (Korolkovas, 1995).

A avaliação da estabilidade acelerada da carbocisteína xarope, visando à previsão do prazo de validade deste produto vem ao encontro da escassez de informações a respeito do mesmo na literatura oficialmente utilizada como referência no Brasil. Através da otimização de metodologia analítica em cromatografia líquida de alta eficiência (Melucci et al., 1987; Tsai et al., 1995; Suntornsuk, 2001) e da aplicação do método de Arrhenius determinou-se o prazo de validade do produto carbocisteína na forma farmacêutica xarope.

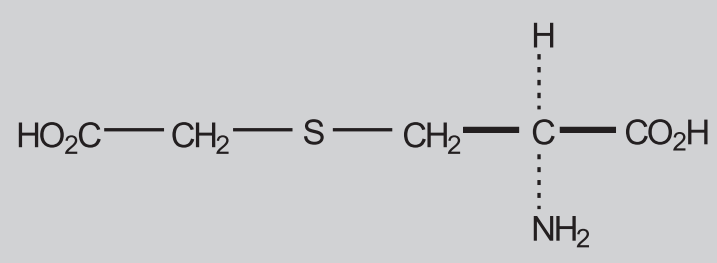

FIGURA 1 - Estrutura química da carbocisteína.

\section{MATERIAL E MÉTODOS}

\section{Substância Química de Referência (SQR)}

Carbocisteína, lote 1a produzido pela Farmacopéia Européia, que foi cedido pela Indústria Farmacêutica Prati, Donaduzzi \& Cia Ltda (Toledo, PR), para realização deste estudo.

\section{Produto Farmacêutico}

Na preparação do xarope, utilizou-se matéria-prima de carbocisteína anidra, produzida pela Moehs Produtos
Químicos, lote 1927, teor 99,84\%, e com prazo de validade até abril de 2008. Como excipientes foram utilizados: sacarina sódica, ácido cítrico, metilparabeno, hidróxido de sódio, ciclamato de sódio, glicerina, álcool etílico, essência de canela, essência de rum, corante caramelo e água desionizada. O xarope foi acondicionado em frascos de $100 \mathrm{~mL}$ de vidro âmbar lacrados com tampas VIC 23 (permeáveis).

\section{Reagentes}

Acetonitrila (Carlo Erba ${ }^{\circledR}$ ), fosfato monobásico de sódio $\left(\right.$ Carlo Erba $\left.^{\circledR}\right)$, água ultrapura $\left(\mathrm{MilliQ}^{\circledR}\right)$.

\section{Metodologia analítica}

A matéria-prima, carbocisteína, foi analisada de acordo com o preconizado na Farmacopéia Européia (2004) e na Farmacopéia Brasileira (1988). Todos os parâmetros avaliados apresentaram-se de acordo com o especificado nestas monografias oficiais (European Pharmacopeia, 2004; Farmacopéia Brasileira, 1988).

O método analítico empregado no doseamento do xarope, por cromatografia líquida de alta eficiência (CLAE) foi validado (Brasil, 2003; ICH, 2006). No doseamento empregou-se cromatógrafo líquido Shimadzu ${ }^{\circledR}$ composto dos seguintes módulos: bomba LC-10AT ${ }_{\mathrm{vp}}$, desgaseificador DGU$14 \mathrm{~A}$, injetor automático SIL-10AF, detector SPD-10A $\mathrm{AP}_{\mathrm{VP}} \mathrm{UV}$ e sistema controlador SCL-10 $\mathrm{A}_{\mathrm{VP}}$. Utilizou-se coluna cromatográfica de fase reversa octadecilsilano $\mathrm{C}_{18}$ Chromolith Performance RP 18-e (100 x 4,6 mm, $5 \mu \mathrm{m})$ MERCK $^{\circledR}$. A fase móvel foi constituída por acetonitrila e solução tampão fosfato monobásico de sódio 10 mM (1:99) no fluxo de $1,5 \mathrm{~mL} / \mathrm{min}$. A detecção foi realizada em $240 \mathrm{~nm}$. A concentração de trabalho foi $2 \mathrm{mg} / \mathrm{mL}$.

As amostras de xarope foram analisadas no tempo zero e dispostas em estufas Quimis ${ }^{\circledR}$ nas condições $40^{\circ} \mathrm{C}$, avaliadas em $72,81,90,100$ e 120 dias; $50^{\circ} \mathrm{C}$, analisadas em $12,24,54,72,84$ e 96 dias; $60^{\circ} \mathrm{C}$, avaliadas em 3, 6, $12,18,27$ e 30 dias e $70^{\circ} \mathrm{C}$, analisadas nos períodos 3,5 , $9,11,15$ e 21 dias. Três frascos de xarope foram analisados em duplicata, em cada tempo e a cada temperatura de armazenamento. O cronograma de retirada de amostras foi rigorosamente cumprido. As amostras retiradas da estufa foram estabilizadas à temperatura ambiente e imediatamente analisadas.

Os resultados de teor, obtidos no estudo de estabilidade, foram avaliados pelo método de Arrhenius a fim de se determinar o prazo de validade do produto em estudo. 


\section{RESULTADOS E DISCUSSÃO}

\section{Validação Analítica}

O método de análise da carbocisteína xarope foi validado através da determinação dos seguintes parâmetros: linearidade, precisão, exatidão, limite de detecção, limite de quantificação, robustez e especificidade de acordo com o preconizado pelo ICH e legislação brasileira (ICH, 2006; BRASIL, 2003). O coeficiente de determinação $\left(\mathrm{r}^{2}\right)$ foi maior que 0,9999 indicando significativa linearidade da curva analítica deste método. O método apresentou-se preciso e exato, com $\mathrm{CV} \%$ de 1,01 e taxa de recuperação de $100,15 \%$. Os limites de quantificação e de detecção obtidos foram de $110,35 \mu \mathrm{g} / \mathrm{mL}$ e $36,41 \mu \mathrm{g} / \mathrm{mL}$, respectivamente. O método apresentou-se robusto mesmo quando submetido a alterações arbitrárias de detecção (comprimento de onda), concentração, fluxo e $\mathrm{pH}$ da fase móvel bem como diferentes lotes de coluna cromatográfica. Quanto à especificidade, a avaliação de amostras de placebo e do xarope submetido à temperatura de $70^{\circ} \mathrm{C}$ não apresentou interferência na análise da carbocisteína.

A Tabela I apresenta a ANOVA utilizada para avaliar os resultados obtidos na elaboração da curva padrão do método apresentado. Verifica-se que não existe desvio significativo da linearidade dos dados utilizados para uma probabilidade menor ou igual a 0,05 .

\section{Estudo de Estabilidade}

As amostras submetidas à degradação térmica foram avaliadas quanto às propriedades organolépticas, $\mathrm{pH}$, teor de carbocisteína e presença de contaminantes microbiológicos. Sob as condições anteriormente citadas o produto apresentou alteração de cor, com mudança da cor amarela original para o castanho escuro e alteração do odor original de canela e rum para um odor desagradável. A alteração de cor pode ser visualizada através da Figura 3, que compara amostra produzida em agosto de 2001 (Laboratório Prati, Donaduzzi \& Cia Ltda), mantida a $25{ }^{\circ} \mathrm{C}$

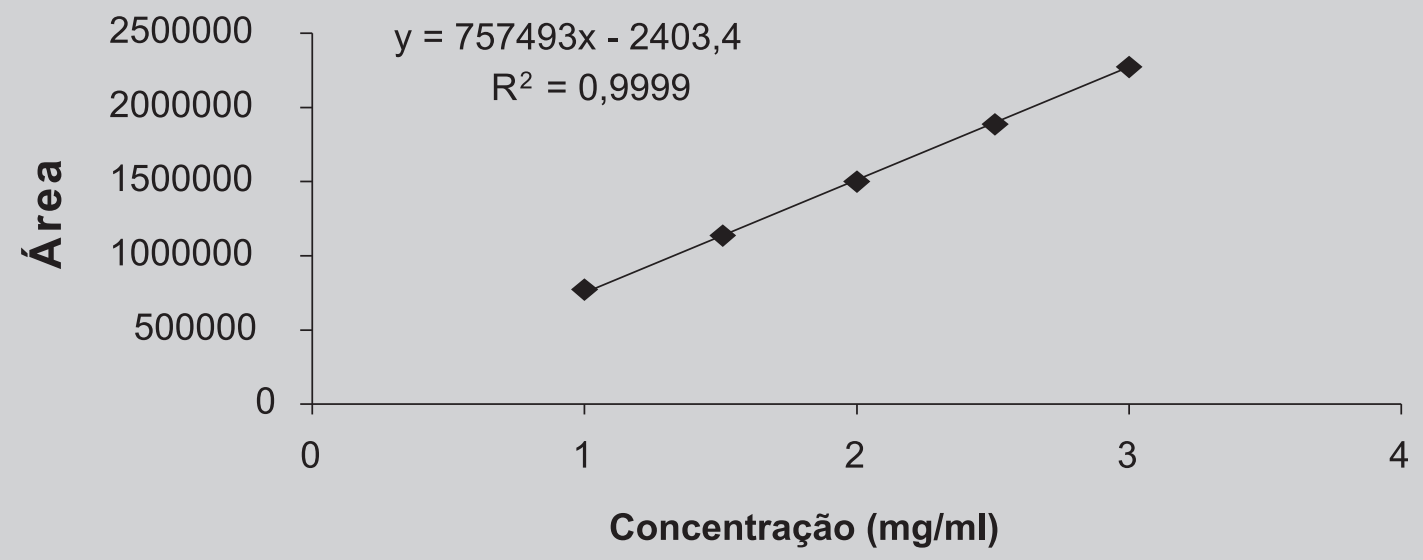

FIGURA 2 - Representação gráfica da curva padrão de carbocisteína obtida por CLAE em fase reversa, com detecção em $240 \mathrm{~nm}$.

TABELA I - Análise de variância dos dados determinados para construção da curva padrão de carbocisteína, utilizando método por CLAE em fase reversa com detecção em $240 \mathrm{~nm}$

\begin{tabular}{lcccc}
\hline Fontes de Variação & gl & SQ & QM & F \\
\hline Entre & 4 & $4,304 \mathrm{E}+12$ & $1,076 \mathrm{E}+12$ & 15,371339 \\
Regressão & 1 & $4,303 \mathrm{E}+12$ & $4,303 \mathrm{E}+12$ & $61,478137^{*}$ \\
Desvio & 3 & 505409645 & 168469882 & 0,0024067 \\
Dentro & 10 & $7 \mathrm{E}+11$ & $7 \mathrm{E}+10$ & \\
Total & 14 & $4,304 \mathrm{E}+12$ & & \\
\hline
\end{tabular}

* significativo para $\mathrm{p}<0,05$ 
(amostra de referência futura), amostra produzida em março de 2004, pelo mesmo laboratório, e, por último, amostra submetida a $50{ }^{\circ} \mathrm{C}$ durante 96 dias.

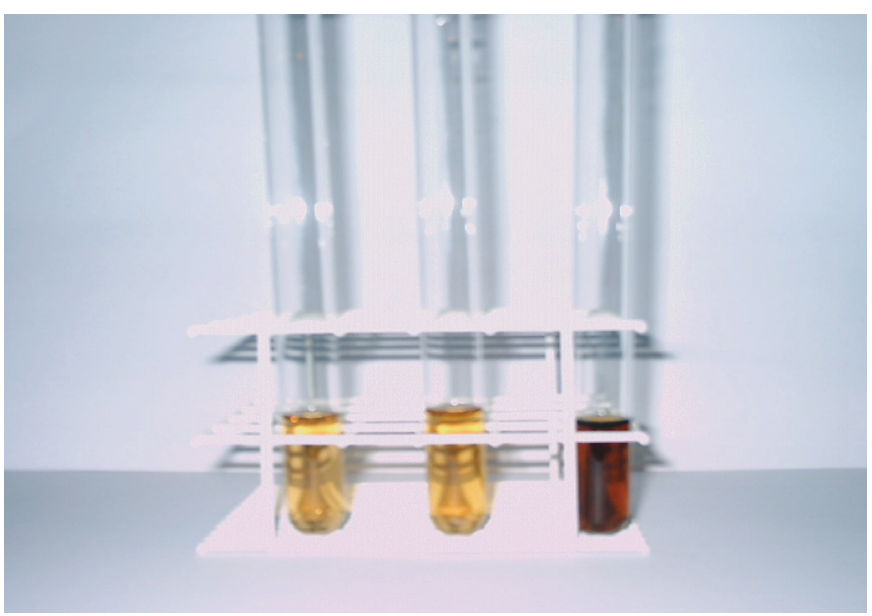

FIGURA 3 - Alteração de cor (da esquerda para a direita): carbocisteína xarope (amostra de referência futura); carbocisteína xarope (mar/04); carbocisteína xarope submetida a $50{ }^{\circ} \mathrm{C}$ durante 96 dias.

O produto foi avaliado quanto à presença de contaminantes microbiológicos de acordo com metodologia farmacopéica (Farmacopéia Brasileira, 1988). As amostras analisadas não apresentaram contaminação microbiológica. Este dado comprova a eficiência do conservante utilizado na formulação.

Os valores indicados na Tabela II correspondem à análise do $\mathrm{pH}$ das amostras submetidas a 40, 50, 60 e $70^{\circ} \mathrm{C}$, obtidos no tempo zero e no último período analisado.

TABELA II - Avaliação do pH das amostras de xarope de carbocisteína sob condições drásticas

\begin{tabular}{cccc}
\hline $\begin{array}{c}\text { Temperatura } \\
\left({ }^{\circ} \mathrm{C}\right)\end{array}$ & $\begin{array}{c}\text { Tempo } \\
\text { (dias) }\end{array}$ & $\begin{array}{c}\mathrm{pH} \\
\text { inicial }^{*}\end{array}$ & $\begin{array}{c}\mathrm{pH} \\
\text { final* }\end{array}$ \\
\hline 40 & 120 & $5,63 \pm 0,00$ & $5,44 \pm 0,02$ \\
50 & 96 & $5,63 \pm 0,00$ & $5,77 \pm 0,07$ \\
60 & 30 & $5,63 \pm 0,00$ & $5,52 \pm 0,01$ \\
70 & 21 & $5,63 \pm 0,00$ & $5,75 \pm 0,02$ \\
\hline
\end{tabular}

*valor médio \pm desvio padrão obtido para $\mathrm{n}=3$.

O doseamento da carbocisteína foi realizado através de método analítico por CLAE previamente validado. As amostras foram analisadas em duplicata, totalizando seis determinações para cada tempo. Os dados estão ilustrados na Tabela III. Os resultados de cada amostra correspondem à média da determinação em duplicata. Foi calculada a média das três amostras, apresentando o resultado em porcentagem e concentração de carbocisteína em mg/mL.

TABELA III - Teor de carbocisteína nas amostras em estudo de estabilidade.

\begin{tabular}{cccc}
\hline $\begin{array}{c}\text { Temperatura } \\
\left({ }^{\circ} \mathrm{C}\right)\end{array}$ & $\begin{array}{c}\text { Tempo } \\
(\text { dias })\end{array}$ & $\begin{array}{c}\text { Concentração } \\
\text { inicial }(\%)^{*}\end{array}$ & $\begin{array}{c}\text { Concentração } \\
\text { final }(\%)^{*}\end{array}$ \\
\hline 40 & 120 & $104,68 \pm 0,04$ & $57,16 \pm 1,96$ \\
50 & 96 & $104,68 \pm 0,04$ & $86,14 \pm 0,60$ \\
60 & 30 & $108,36 \pm 0,23$ & $61,80 \pm 3,92$ \\
70 & 21 & $102,70 \pm 1,19$ & $43,98 \pm 2,09$ \\
\hline
\end{tabular}

*valor médio \pm desvio padrão obtido para $\mathrm{n}=3$.

\section{Determinação do prazo de validade}

A partir dos resultados obtidos na análise de teor de carbocisteína nas amostras submetidas às condições drásticas, foram elaborados gráficos para cada temperatura, visando determinar a ordem da reação de degradação: concentração versus tempo; $\log$ da concentração versus tempo e inverso da concentração versus tempo.

Os valores obtidos das inclinações das retas (k') dos gráficos do log da concentração versus tempo foram utilizados para determinar o prazo de validade do produto, pois apresentaram os melhores valores de $\mathrm{R}^{2}$, ou seja, valor médio de 0,9457 . Os dados relativos às amostras acondicionadas a $50{ }^{\circ} \mathrm{C}$ foram excluídos devido à baixa degradação da carbocisteína durante o período de armazenamento. A tabela IV exibe os valores de k' para cada temperatura.

TABELA IV - Valores de k' observados nos gráficos do log da concentração versus tempo de cada temperatura avaliada

\begin{tabular}{cc}
\hline Temperatura $\left({ }^{\circ} \mathrm{C}\right)$ & $\mathrm{k}^{\prime}$ \\
\hline 40 & 0,0019 \\
60 & 0,0076 \\
70 & 0,0186 \\
\hline
\end{tabular}

A equação de Arrhenius foi empregada para determinar a energia de ativação $(\Delta \mathrm{E})$ nas diferentes temperaturas avaliadas.

$$
\log \mathrm{k}_{2} / \mathrm{k}_{1}=\Delta \mathrm{E} / 2,303 \mathrm{R} \times\left(\mathrm{T}_{2}-\mathrm{T}_{1} / \mathrm{T}_{2} \times \mathrm{T}_{1}\right)
$$

onde R: 1,987 (constante dos gases) e T: temperatura em graus Kelvin

A partir da média da energia de ativação obtida $(\Delta \mathrm{E})$ 
foi determinada a constante de degradação a $25^{\circ} \mathrm{C}\left(\mathrm{k}_{25}\right)$ através da equação de Arrhenius. A constante $\mathrm{k}_{1}$ foi substituída por uma das constantes de degradação k, previamente calculadas. $\mathrm{O}$ valor $\mathrm{de} \mathrm{k}_{25}$ foi determinado com base nas constantes de degradação a 40, 60 e $70^{\circ} \mathrm{C}$.

$$
\begin{gathered}
\Delta \mathrm{E} \text { médio }=16.943,56 \\
\mathrm{k}_{25} \text { médio }=0,00044
\end{gathered}
$$

O prazo de validade da carbocisteína xarope foi calculado a partir da cinética de degradação. No caso de uma reação de primeira ordem (pseudo-primeira ordem), o prazo de validade pode ser determinado através do cálculo do $\mathrm{T}_{90 \%}$ segundo a equação descrita na seqüência.

$$
\mathrm{T}_{90 \%}=0,106 / \mathrm{k}_{25}
$$

$\mathrm{O}$ valor de $\mathrm{T}_{90 \%}$ calculado a partir da média dos $\mathrm{k}_{25}$ determinados com base nas constantes de degradação obtidos a 40,60 e $70^{\circ} \mathrm{C}$ foi de 240,90 dias, aproximadamente 8 meses.

\section{Cromatogramas de amostras sob condições drásticas}

Nos cromatogramas relativos às condições de $60 \mathrm{e}$ $70{ }^{\circ} \mathrm{C}$ foi detectado um pico posterior ao pico principal da carbocisteína. Desta forma, com base nas informações relativas à rota de síntese deste composto, buscou-se identificar o pico de degradação observado. A síntese da carbocisteína emprega a L-cisteína, constituída por duas moléculas de cistina. Ambas as substâncias foram analisadas através do método empregado na análise da carbocisteína para verificar se o tempo de retenção seria igual ao do produto de degradação. De acordo com a Figura 4, o produto de degradação exibido no cromatograma abaixo não corresponde a L-cistina ou a L-cisteína, cujos cromatogramas podem ser visualizados nas Figuras 5 e 6 , respectivamente, cujas amostras foram preparadas nas mesmas condições descritas no método de análise da carbocisteína xarope.

\section{CONCLUSÕES}

O método analítico utilizado na determinação da carbocisteína em xaropes apresentou-se linear, preciso,

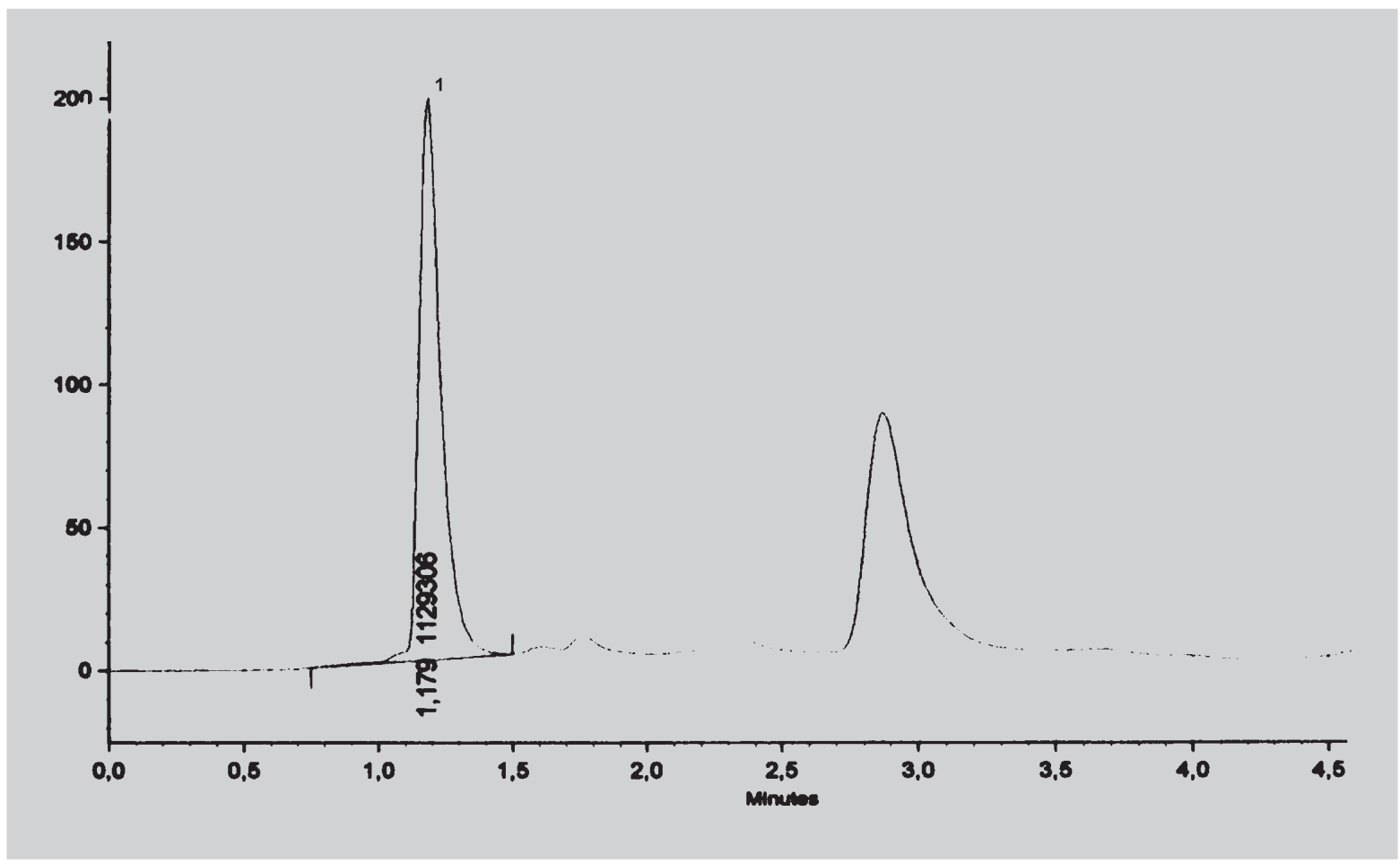

FIGURA 4 - Cromatograma da carbocisteína (1) xarope exposta à temperatura de $70^{\circ} \mathrm{C}$. Fase móvel ACN:Tampão fosfato

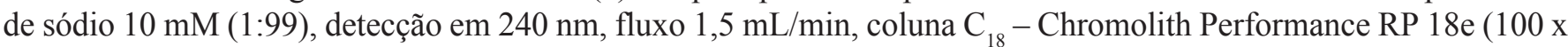
$4,6 \mathrm{~mm}, 5 \mu \mathrm{m})$. 


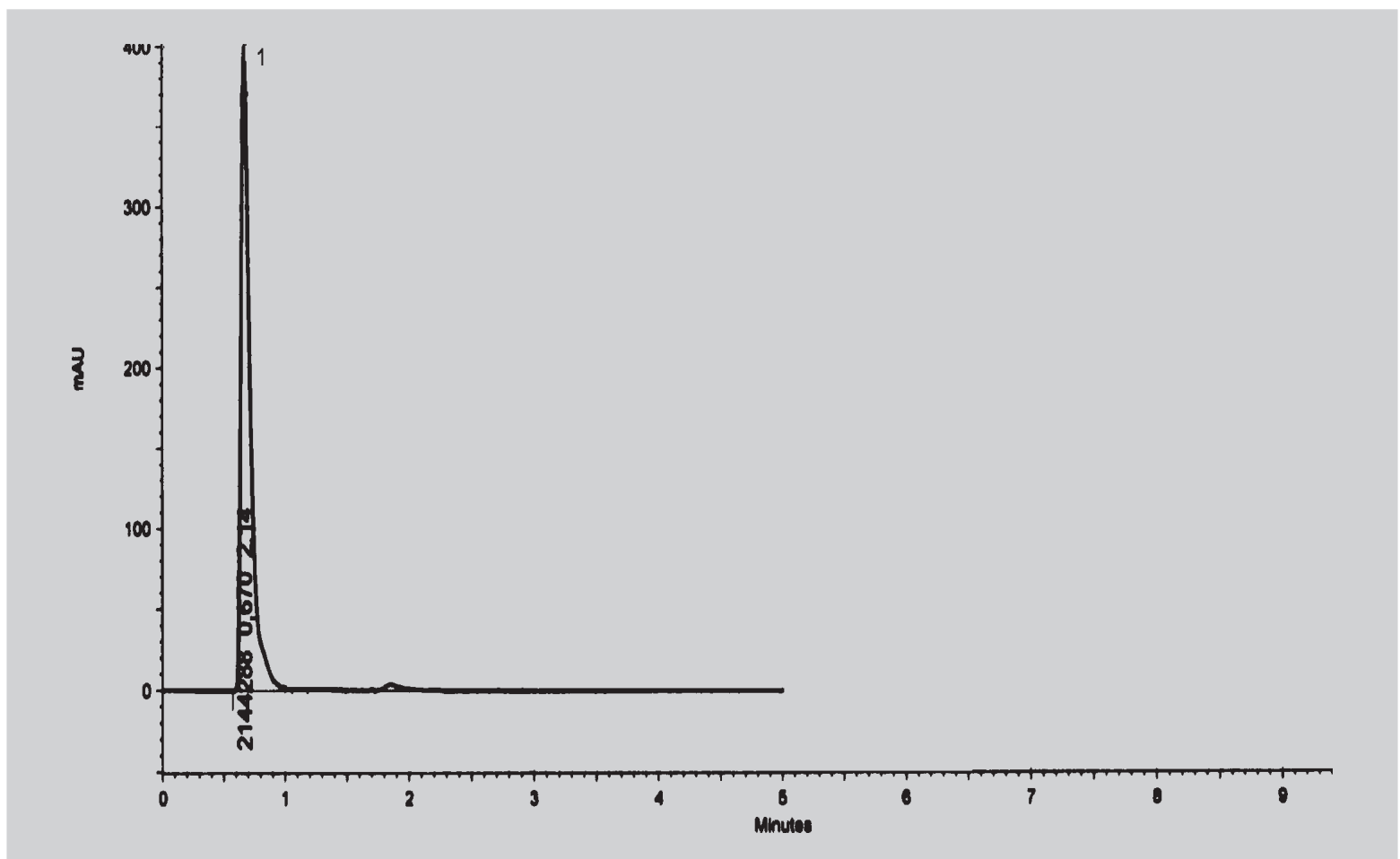

FIGURA 5 - Cromatograma da L-cistina susbtância de referência (Sigma $\left.{ }^{\circledR}\right)$.Concentração de L-cistina $2 \mathrm{mg} / \mathrm{ml}$, fase móvel ACN:tampão fosfato de sódio $10 \mathrm{mM}$ (1:99), detecção em $240 \mathrm{~nm}$, fluxo 1,5 mL/min, coluna $\mathrm{C}_{18}-$ Chromolith Performance RP 18e (100 x 4,6 mm, $5 \mu \mathrm{m})$.

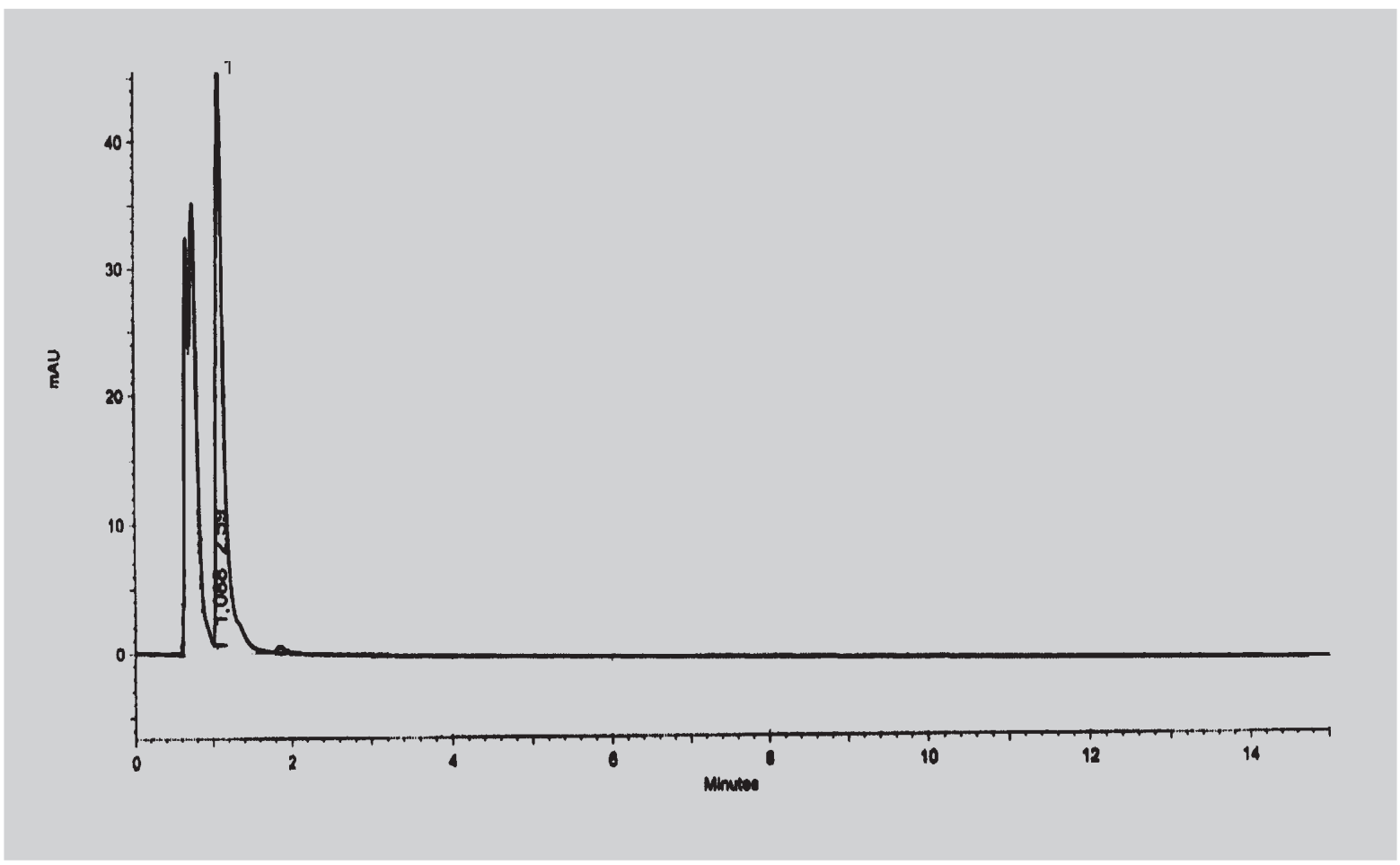

FIGURA 6 - Cromatograma da L-cisteína substância de referência $\left(\right.$ Sigma $\left.^{\circledR}\right)$. Concentração 2 mg/ml, fase móvel ACN:tampão fosfato de sódio $10 \mathrm{mM}$ (1:99), detecção em $240 \mathrm{~nm}$, fluxo 1,5 mL/min, coluna $\mathrm{C}_{18}-$ Chromolith Performance RP 18e (100 x 4,6 mm, $5 \mu \mathrm{m})$. 
exato, específico e robusto, permitindo a correta determinação deste fármaco em ensaios de rotina no controle de qualidade ou em estudos de estabilidade.

A carbocisteína xarope apresenta alteração significativa no teor do fármaco quando exposta às temperaturas elevadas. Simultaneamente ocorreram alterações na cor e odor da preparação em conseqüência de alterações do corante e essência utilizados na formulação, respectivamente.

Nos cromatogramas analíticos observou-se a presença de produto de degradação. Foi investigada a possibilidade desta substância tratar-se de L-cisteína ou L-cistina, ambas precursoras da carbocisteína. No entanto, estas não apresentavam o mesmo tempo de retenção observado no produto de degradação em temperaturas elevadas.

A cinética de degradação da carbocisteína corresponde a uma reação de pseudo-primeira ordem.

O prazo de validade para a formulação estudada é de 240,90 dias, o que não corresponde à validade de dois anos dos produtos à base de carbocisteína comercializados no Brasil.

Por fim, conclui-se que o estudo de estabilidade acelerado é uma ferramenta útil na previsão do prazo de validade de produtos farmacêuticos. No entanto, faz-se necessária a avaliação do produto através do estudo de estabilidade de Longa Duração, desta forma determinando a validade definitiva do mesmo.

\section{AGRADECIMENTOS}

Este estudo foi patrocinado pela Indústria Farmacêutica Prati, Donaduzzi \& Cia LTDA -Toledo-PR.

\section{ABSTRACT}

\section{Determination of carbocysteine syrup shelf life by Arrhenius method}

Carbocysteine is a mucolytic agent in adjunctive therapy of respiratory tract infections. The pharmaceutical industry is responsible for quality, safety and efficiency of the product during its shelf life. Shelf life can be determinated through an accelerated stability study where the degradation of the drug is managed with the extrinsic factors. According to Arrhenius, there is a relationship between temperature and chemical kinetic, so, the samples were exposed to drastic conditions at 40, 50, 60 and $70^{\circ} \mathrm{C}$. The syrup assay method has been validated and it may be applied to analysis of carbocysteine syrup in routine quality control and stability studies. Through Arrhenius equation the proposed shelf life of carbocysteine syrup was 240.9 days when the dosage form is stored in appropriated conditions, $25^{\circ} \mathrm{C}$. However difference was found between the proposed shelf life and the usual shelf life of this drug. In addition, this study has showed the presence of endogenous peaks that must be better evaluated to confirm or not the presence of degradation products.

UNITERMS: Arrhenius method. Carbocisteyne/syrup. Drugs/stability study.

\section{REFERÊNCIAS BIBLIOGRÁFICAS}

BRASIL. Resolução RE n ${ }^{0} 899$, de 29 de maio de 2003. A Agência Nacional de Vigilância Sanitária determina a publicação do "Guia para validação de métodos analíticos e bioanalíticos". Diário Oficial da União. Brasília, 2 jun. 2003.

BRASIL. Resolução RE n 1 , de 29 de julho de 2005. A Agência Nacional de Vigilância Sanitária autoriza ad referendum, a publicação do "Guia para realização de Estudos de Estabilidade”. Diário Oficial da União. Brasília, 01 ago. 2005.

BRASIL. Agência Nacional de Vigilância Sanitária. Banco de Dados. Disponível em <www7.anvisa.gov.br/datavisa/ consulta produto/consulta medicamento.asp $>$. Acesso em: 20 ago. 2007.

BAKSHI, M.; SINGH, S. Development of validated stabilityindicating assay methods-critical review. J. Pharm. Biomed. Anal., v. 28, p.1011-1040, 2002.

EUROPEAN Pharmacopoeia. 4 ed. Strasbourg: European Department for the Quality of Medicines, 2004. p. 1063.

FARMACOPÉIA Brasileira. 4.ed. São Paulo: Atheneu, 19881996. pt.2, p. 1213.

ICH - Steering Committee International Conference on Harmonisation. Geneva: Of Technical Requirements for Registration of Pharmaceuticals for Human Use. Validation of Analytical Procedures: methodology, 2006, p. $1-8$.

KOROLKOVAS, A. Dicionário terapêutico Guanabara. Edição 1995/1996. Rio de Janeiro: Editora Guanabara Koogan, 1995. p. 664. 
LACHMAN, L. LIEBERUAN, H. A.; KANIG, J. L. Teoria e prática na indústria farmacêutica. Lisboa: Fundação Calouse Gulben Kian, 2001. p. 1517.

MELUCCI, C.K.; LYMAN, G.W.; BOND, A.D.; JOHNSON, R.N. Determination of Scarboxymethylcysteine in syrup formulations by highperformance liquid chromatography. J. Chromatogr., v. 391, p.321-324, 1987.

SUNTORNSUK, L. Direct determination of scarboxymethyl-1-cysteine in syrups by reversed-phase high-performance liquid chromatography. J. Pharm. Biomed. Anal., v. 25, p.165-170, 2001.
TSAI, F. Y.; CHEN, C. J.; CHIEN, C. S. Determination of the cysteine derivatives N-acetylcysteine, Scarxymethylcysteine and methylcysteine in pharmaceuticals by high-performance liquid chromatography. J. Chromatogr., v. 697, p.309-315, 1995.

Recebido para publicação em 19 de outubro de 2006. Aceito para publicação em 22 de agosto de 2007. 